\title{
Musculoskeletal adverse reactions after immunotherapy for cancer: A case series
}

\author{
IOANA CREŢU ${ }^{1,2}$, MIHAI BOJINCĂ ${ }^{1,2}$, MIHAELA MILICESCU ${ }^{1,2}$, ADRIAN CURSARU $^{1,3}$, \\ BOGDAN ŞERBAN ${ }^{1,3}$, BOGDAN CREȚU ${ }^{1,3}$, SERGIU IORDACHE ${ }^{1,3}$, CORINA SILVIA POP ${ }^{1,4}$, \\ CĂTĂLIN CÎRSTOIU ${ }^{1,3}$ and RUXANDRA IONESCU ${ }^{1,5}$
}

\begin{abstract}
${ }^{1}$ Department of Internal Medicine and Rheumatology, 'Carol Davila' University of Medicine and Pharmacy, 020021 Bucharest;
${ }^{2}$ Department of Internal Medicine and Rheumatology, 'Dr. Ion Cantacuzino' Hospital, 917151 Bucharest; Departments of ${ }^{3}$ Orthopedics and Traumatology, and ${ }^{4}$ Internal Medicine, University Emergency Hospital, 050098 Bucharest; ${ }^{5}$ Department of Internal Medicine and Rheumatology, 'Sf. Maria' Clinical Hospital, 011172 Bucharest, Romania
\end{abstract}

Received May 10, 2021; Accepted June 9, 2021

DOI: $10.3892 /$ etm.2021.10459

\begin{abstract}
Immunotherapy has revolutionized cancer treatment. Immune checkpoint inhibitors (ICIs) including antibodies targeting cytotoxic $\mathrm{T}$ lymphocyte associated antigen-4 and programmed cell death 1 have been shown to be effective in the treatment of certain types of cancer. The benefit of these therapies is to prolong life expectancy in the case of metastatic malignancies. Rheumatic adverse events are not very common. In the present study, 9 patients were monitored between November 2018 and January 2020. The oncologist, who identified the occurrence of rheumatic toxicities after the treatment with ICIs, evaluated the patients. Only oncological patients with rheumatic manifestations after the start of immunotherapy were included. Toxicity grading was performed by both the oncologist and the rheumatologist, on a scale from 1 to 5 (1, mild; 2 , moderate; 3 , severe; 4 , life-threatening; 5 , death related to toxicity). The results showed that rheumatoid factor, which was sampled in each patient, was negative in all cases. Patients were treated with nonsteroidal anti-inflammatory drugs or prednisone depending on the severity of the adverse events. The results varied with the severity of the adverse events. In conclusion, as the number of patients treated with ICIs increases, so will the number of patients presenting with immune-related adverse events (irAEs). The collaboration between oncologists and rheumatologists should be intimate to provide optimal treatment to patients. Musculoskeletal manifestations secondary to ICIs are slightly different from other rheumatologically conditions making diagnosis, treatment
\end{abstract}

Correspondence to: Dr Bogdan Crețu, Department of Orthopedics and Traumatology, University Emergency Hospital, 169 Splaiul Independentei, 050098 Bucharest, Romania

E-mail: jfrbogdan@yahoo.com

Key words: immunotherapy, musculoskeletal adverse events, cancer, immune checkpoint inhibitors, rheumatology and monitoring difficult. Thus, irAEs are new and challenging for oncologists, thus understanding of the pathogenesis and clinical characteristics must be improved for better treatment guidelines.

\section{Introduction}

Immunotherapy has revolutionized cancer treatment. Immune checkpoint inhibitors (ICIs) including antibodies targeting cytotoxic T lymphocyte associated antigen-4 (CTLA-4) and programmed cell death 1 (PD-1) have been shown to beeffective in the treatment of certain types of cancer, such as melanoma, as well as largecell lung, urothelial, genitourinary and gastrointestinal cancers. The benefit of these therapies is to prolong life expectancy in the case of metastatic malignancies $(1,2)$.

Immunotherapy has an effective antitumor effect, but it modifies immunological homeostasis by blocking the negative regulators of the immune system, which leads to the appearance of immunological adverse events (3). ICIs act on the level of inhibitory pathways at different stages. CTLA-4 acts in the initial stage, preventing the $\mathrm{T}$ cells from responding after presentation of the tumor antigen. The second ICI is PD-1, an activated effector of cytotoxic T cells. PD-1 binds to ligand 1 or 2 (PD-L1, PD-L2) which are exposed in the tumor cells and lead to apoptosis. Anti-PD-1 or anti-PD-2 acts at this stage, disrupting immune tolerance and leading to the ability of T cells to attack the tumor cell $(4,5)$. Considering the different functions of CTLA-4 and PD-1, different side effects can be highlighted. Anti-CTLA-4 toxicities are more severe, hypophysitis being the most common (6). In patients treated with anti-PD-1, pulmonary side effects and thyroiditis occur more frequently $(7,8)$.

Currently, six molecules of ICIs have been approved by the Food and Drug Administration (FDA) and are used for the treatment of solid and hematologic tumors (9). Ipilimumab is a complete human monoclonal antibody IgG1 that blocks CTLA-4; it was the first one approved in 2011 for the treatment of metastatic melanoma. Pembrolizumab and nivolumab are humanized monoclonal antibodies acting against receptor 1, 
with a role in controlling programmed cell death (PD-1, programmed cell death-1), which received FDA approval in $2014(10,11)$. The indications of these two molecules have expanded greatly. In 2015, nivolumab was approved for the treatment of large cell lung cancer. Later in 2015, the best combination of ipilimumab and nivolumab against melanoma was approved. Recently, three new molecules have been approved, namely atezolizumab, durvalumab and avelumab. All three molecules act against ligand 1 with a role in controlling programmed cell death (PD-L1) $(10,12)$. Atezolizumab and durvalumab are humanized IgG1 monoclonal antibodies modified in the Fc region, while avelumab has the unchanged Fc portion. Since 2016, atezolizumab and durvalumab have been approved for the treatment of large cell lung cancer and for urothelial cancer, and avelumab has been approved for Merkel cell carcinoma and urothelial cancer $(2,13)$.

As the number of patients treated with ICIs increases, so does the number of adverse events (irAEs, immune-related adverse events) (14). The toxicities that occur after using these treatments can affect any organ or tissue, but some irAEs occur more frequently than others. The skin, colon, endocrine organs, liver, and lung are most commonly affected (15). Other toxicities may appear rarely and cause important adverse events, such as myocarditis or neurological disorders. Generally, adverse events occur within the first weeks up to 3 months after initiating treatment with ICIs. However, adverse events have been reported 1 year after the initiation of therapy $(1,10)$.

Prior to starting treatment, patients should be examined, in order to highlight their predisposition of developing irAEs (7). Thus, it is important to know the medical history of the patient, as well as the family history, history of autoimmune diseases, basic laboratory tests, and also radiological investigations. Patients who have a medical history of autoimmune diseases or those who are treated for an autoimmune disease are prone to a negative evolution while being treated with ICIs $(9,14)$. Once adverse events have occurred, rapid intervention is needed to prevent exacerbation of symptoms. In many cases, especially in severe cases, immunotherapy should be discontinued and treatment with immunosuppressive drugs should be started. The drugs used to combat toxicities are high doses of corticosteroids and sometimes anti-TNF- $\alpha$, mycophenolate and tacrolimus (16).

Rheumatic adverse events are not very common. The incidence of rheumatic manifestations after ICIs is not really known $(8,9)$. There are two main reasons why there is no proper evidence of musculoskeletal adverse events. First, the evaluation of the rheumatic adverse events in oncological studies is incorrect. For example, arthritis is coded as arthralgia, arthritis, or musculoskeletal pain. The second reason is the system of grading of the adverse events used in oncological studies (12). Most studies do not report adverse events greater than 3 rd degree, the 5 th degree being the highest of severity (1, mild; 2 , moderate; 3 , severe; 4 , life-threatening; 5 , death related to toxicity). However, severe adverse events (3rd-5thdegree) that require hospitalization are quite rare (2).

\section{Patients and methods}

Patients. Nine oncological patients with rheumatic manifestations after the start of immunotherapy were included in the present study. Inclusion criteria were: Oncological patients in treatment with ICIs and with rheumatic adverse events. The 9 patients, 8 men and 1 woman, aged $\geq 18$ years (mean age, 61 years), were monitored between November 2018 and January 2020.

Approval of the study (no. 40103/13.08.2020) was obtained from the Internal Medicine, University Emergency Hospital, 050098 Bucharest, Romania. Written informed consent was obtained from all 9 patients.

Patient evaluation. The oncologist, who identified the occurrence of rheumatic toxicities after the treatment with ICIs, evaluated the patients. Toxicity grading was performed by both the oncologist and the rheumatologist based on the general terminology standard for adverse events as per the Common Terminology Criteria for Adverse Events (CTCAE), on a scale from 1 to 5 (1, mild; 2 , moderate; 3 , severe; 4, life-threatening; 5 , death related to toxicity) (17).

The 9 patients received treatment with ipilimumab, pembrolizumab and atezolizumab for metastatic melanoma, non-small cell lung cancer and urothelial carcinoma. The rheumatologist who performed the clinical examination of the painful joints, tracking of the presence of joint swelling, impaired joint function, and morning stiffness, also evaluated the patients. Musculoskeletal ultrasound was performed at the level of the painful joints revealing synovial effusion and synovial proliferation. The ultrasound protocol used for evaluation was carried out according to the European League against Rheumatism (EULAR) guidelines, using gray scale and power Doppler (18). After the evaluation, rheumatic manifestations, such as inflammatory arthritis, arthralgia, myalgia, and rheumatoid arthritis were included. The differential diagnosis for other pathologies that can cause joint manifestations (i.e.,osteoarthritis, or polymyalgia rheumatica) was performed by combining clinical examination, ultrasound check-up and by biological findings.

The following information was entered into the database: Age, sex, type of malignancy, immunotherapy (type of medication and duration of treatment until the occurrence of adverse events), C-reactive protein (CRP), erythrocyte sedimentation rate (ESR), rheumatoid factor (RF), irAE treatment, response to treatment, grade of irAEs, and whether discontinuation of the oncological treatment was required. The response to treatment was evaluated as positive in patients who had no musculoskeletal pain at all or had mild pain that did not require treatment. Anegative response was marked in patients who suffered from continuous joint pain after discontinuing the rheumatic treatment.

\section{Results}

Demographic data and oncological history. Nine patients were evaluated, of whom 8 were men and 1 woman. The mean age of the patients was 61 years. Five patients received nivolumab, 3 patients received pembrolizumab and 1 patient received atezolizumab. Four patients were diagnosed with metastatic melanoma, 3 with non-small cell lung cancer, 1 patient with urothelial carcinoma, and 1 patient with renal carcinoma.

The time at which the rheumatic toxicities occurred after the beginning of the treatment with ICIs was an average of 10 weeks (2-20 weeks). 
Rheumatic toxicities occurring after ICIs. The clinical presentation of the patients was varied (Table I). Pain appeared both at the large and small joint levels. Shoulder pain occurred in 2 patients, of whom 1 patient had bilateral subacromial-subdeltoid (SASD) bursitis and bilateral bicipital tendinitis. Only 1 patient had elbow pain. Knee pain occurred in 5 of the 9 patients, and 2 of these 5 patients presented with synovial fluid in medium quantity at the level of bilateral suprapatellar recess. Four patients had pain in the metacarpophalangeal and proximal interphalangeal joints. Only 1 patient had small joint effusion, being diagnosed with seronegative rheumatoid arthritis. Two patients had major myalgia, without having the muscle enzymes modified. Myalgia appeared in the upper and lower limbs, and lof the patients also had extrapyramidal syndrome associated with muscle pain.

RF was sampled in all the patients, and was negative for all 9 cases. Markers of inflammation, ESR and CRP were sampled, and the values were elevated. The values of ESR and CRP can be elevated in the context of malignancy and thus are unable to be good indicators for joint damage. However, the highest values of ESR and CRP appeared in patients with the most severe impairment.

IrAE grading was performed according to joint damage. Three patients were classified as 1st degree, i.e., mild impairment. Three patients were included in the 2nd degree category, i.e., moderate impairment, and another 3 patients were included in the 3rd degree category, i.e., severe impairment. In the case of those with severe illness, treatment with ICIs was interrupted, in 2 of the patients the decision being final. In 1 patient, the treatment with ICIs was resumed after improvement of the joint symptoms.

Musculoskeletal adverse events occurred on average at 10 weeks after initiating treatment with ICIs. One patient had toxicities 2 weeks after the first administration. In another patient, rheumatic diseases appeared 20 weeks after the first administration, but before the onset of symptoms, of a double dose of nivolumab.

Treatment of rheumatic adverse events. Patients were treated with nonsteroidal anti-inflammatory drugs (NSAIDs) or prednisone depending on the severity of the adverse events. One patient was diagnosed with seronegative rheumatoid arthritis after treatment with ICIs and was treated with dexamethasone for 5 days and then started treatment with sulphasalazine 2 g/day.

Another patient who experienced severe adverse events after increasing the dose of nivolumab received dexamethasone for 5 days with mild improvement in symptomatology and then received treatment with prednisone at $15 \mathrm{mg} / \mathrm{day}$.

Patients with mild rheumatoid toxicity received NSAID treatment, with symptom relief improving after approximately 2 weeks. Patients with moderate adverse events were treated with prednisone $10 \mathrm{mg} / \mathrm{day}$, with a gradual decrease of the dose after 2 weeks until corticotherapy was stopped. In the case of severe adverse events, treatment with ICIs was discontinued and high-dose prednisone treatment was initiated. Of the 3 patients with severe events, only 1 patient resumed treatment with ICIs. Another patient did not continue oncological treatment due to tumor progression under ICIs, but also because of persistent joint pain.

\section{Discussion}

As the number of patients treated with immune checkpoint inhibitors (ICIs) increase, so do the number of patients with immune-related adverse events (irAEs); the collaboration between oncologists and rheumatologists should be therefore closer in order to treat these patients correctly (8).

In the present study, 9 patients who had rheumatic toxicities were identified. Of these, 8 patients were treated with anti-PD-1 and 1 patient with anti-PD-L1. The average time of adverse events was approximately 10 weeks, in other studies being approximately 12 weeks (3). Some patients have symptoms after discontinuation of ICIs.

Regarding the treatment, patients responded well to NSAID treatment and corticosteroid therapy. From the existing data, the symptomatology was expected to disappear in 6-12 weeks of corticosteroid therapy. In severe cases, biological therapy is considered the treatment of choice (18). However, such options are not approved in Romania. In addition, patients who do not respond to corticosteroid therapy cannot be treated with more aggressive immunosuppression.

There are several limitations to the current study. The number of patients was not high, only 9 patients during a year. This is due to the small number of patients treated with ICIs in Romania, but also because of the lack of close collaboration between oncologists and rheumatologists. Conversely, rheumatologists examine only patients sent by the oncologists, without knowing exactly the incidence of irAEs.

The evaluated patients in the present study received treatment with nivolumab, pembrolizumab and atezolizumab; in addition, there was not much diversity of ICIs. In patients with inflammatory arthritis, no joint fluid analysis was performed. The diagnosis was made after clinical, ultrasound and biological evaluation.

Musculoskeletal manifestations secondary to ICIs were slightly different from other rheumatological conditions; for example, inflammatory arthritis secondary to ICIs did not have the typical characteristics as conventional rheumatoid arthritis.

In conclusion, the real incidence of rheumatic adverse events is not known, even if there are risk factors for the development of toxicity when ICIs are used $(3,8,19)$. The clinical, ultrasound and biological presentation of the patients with rheumatoid toxicities is varied, and a thorough examination is needed in order to correctly diagnose these patients. Currently, patients are treated by rheumatologists based on their clinical experience, the latter collaborating with oncologists for the optimal therapeutic decision as it is crucial to have a common treatment guide.

The use of ICIs has led to the incidence of irAEs, creating new challenges for the oncologists. Presently, irAEs are classified according to the CTCAE and managed according to international protocols, which include NSAIDs, glucocorticosteroids and disease-modifying antirheumatic drugs. For the oncologists, rheumatic manifestations are new. Therefore, understanding the pathogenesis and clinical characteristics and treatments must be improved in order to develop therapeutic strategies. 


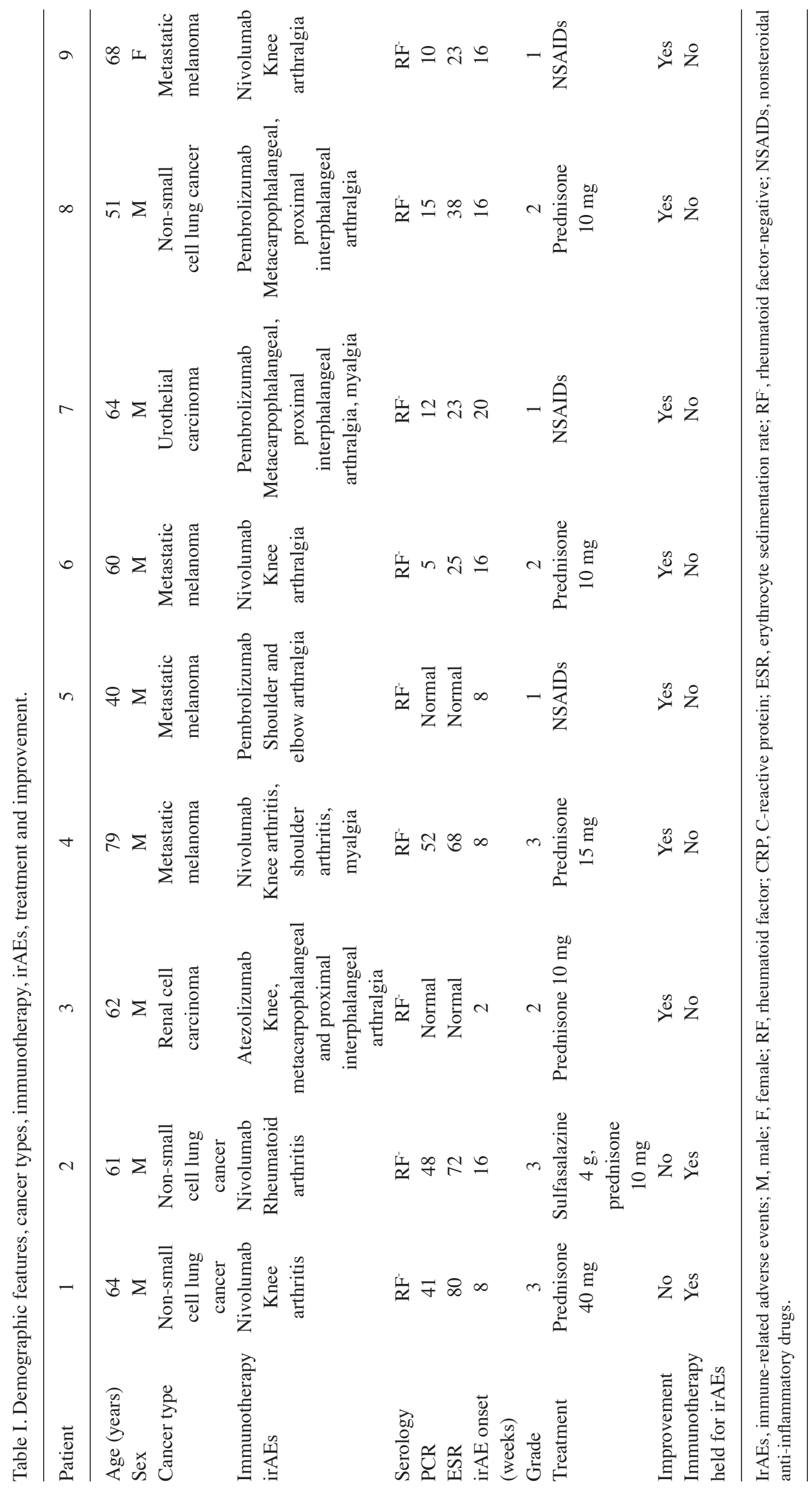




\section{Acknowledgements}

Professional editing, linguistic and technical assistance performed by Irina Radu, Individual Service Provider.

\section{Funding}

No funding was received.

\section{Availability of data and materials}

Further findings concerning the study can be obtained by contacting the Corresponding author.

\section{Authors' contributions}

IC, $\mathrm{MB}$ and $\mathrm{MM}$ were responsible for conceiving and designing the study. AC, SI and BS carried out data acquisition and performed analysis of the data. BC and CSP conducted interpretation of the data, wrote and revised the manuscript. $\mathrm{CC}$ and RI reviewed and edited the manuscript. IC, BC and MM confirm the authenticity of all the raw data. All authors read and approved the final manuscript for publication.

\section{Ethics approval and consent to participate}

Approval of the study (no. 40103/13.08.2020) was obtained from the Internal Medicine, University Emergency Hospital, 050098 Bucharest, Romania. Written informed consent was obtained from all 9 patients.

\section{Patient consent for publication}

Not applicable.

\section{Competing interests}

The authors declare that they have no competing interests.

\section{References}

1. Cappeli LC, Shah AA and Bingham CO III: Immune-related adverse effects of cancer immunotherapy-Implications for rheumatology. Rheum Dis Clin North Am 43: 65-78, 2017.

2. Robert C, Schachter J, Long GV, Arance A, Grob JJ, Mortier L, Daud A, Carlino MS, McNeil C, Lotem M, et al: Pembrolizumab versus ipilimumab inadvanced melanoma. N Eng J Med 372 $2521-2532,2015$

3. Lidar M, Giat E, Garelick D, Horowitz Y, Amital H, Steinberg-Siliman Y, Schachter J, Saphira-Frommer R and Markel G: Rheumatic manifestation among cancer patients treated with immune checkpoint inhibitors. Autoimmun Rev 17: 284-289, 2018
4. Jenkins WR, Barbie DA and Flaherty KT: Mechanisms of resistance to immune checkpoint inhibitors. Br J Cancer 118: 9-16, 2018.

5. Pardoll DM: The blockade of immune checkpoints in cancer immunotherapy. Nat Rev Cancer 12: 252-264, 2012.

6. Moreira A, Loquai C, Pfohler C, Kähler KC, Knauss S, Heppt MV, Gutzmer R, Dimitriou F, Meier F, Mitzel-Rink H, et al: Myositis and neuromuscular side-effects induced by immune checkpoint inhibitors. Eur J Cancer 106: 12-23, 2019

7. Alias ADO, Rodriguez EJ, Bateman HB, Sterrett AG and Valeriano-Marcet J: An updated review of rheumatic manifestations of malignancy and anti-neoplastic therapy. Bull NYU Hosp Jt Dis 70: 109-114, 2012.

8. Cappelli LC, Gutierrez AK, Baer AN, Albayda J, Manno R, Haque U, Lipson EJ, Bleich KB, Shah AA, Naidoo J, et al: Inflammatory arthritis and sicca syndrome induced by nivolumab and ipilimumab. Ann Rheum Dis 76: 43-50, 2017.

9. Haanen JBAG, Carbonnel F, Robert C, Kerr KM, Peters S, Larkin $\mathrm{J}$ and Jordan $\mathrm{K}$; ESMO Guidelines Committee: Management of toxicities from immunotherapy: ESMO Clinical Practice Guidelines for diagnosis, treatment and follow-up. Ann Onco 29 (Suppl 4): iv264-iv266, 2018.

10. Kyung-Ann L, Hae-Rim K and So Young Y: Rheumatic complications in cancer patients treated with immune checkpoint inhibitors. Korean J Intern Med 34: 1197-1209, 2019.

11. Blansfiield JA, Beck KE, Tran K, Yang JC, Hughes MS, Kammula US, Royal RE, Topalian SL, Haworth LR, Levy C, et al: Cytotoxic T-lymphocyte-associated antigen-4 blockage can induce autoimmune hypophysitis in patients with metastatic melanoma and renal cancer. J Immunother 28: 593-598, 2005.

12. Kyi $\mathrm{C}$ and Postow MA: Checkpoint blocking antibodies in cancer immunotherapy. FERBS Lett 588: 368-376, 2014.

13. Menzies AM, Johnson DB, Ramanujam S, Atkinson VG, Wong ANM, Park JJ, McQuade JL, Shoushtari AN, Tsai KK, Eroglu Z, et al: Anti-PD-1 therapy in patients with advanced melanoma and preexisting autoimmune disorders or major toxicity with ipilimumab. Ann Oncol 28: 368-376, 2017.

14. Kumar V, Chaudhary N, Garg M, Floudas CS, Soni P and Chandra AB: Current diagnosis and management of immune related adverse events (irAEs) induced by immune checkpoint inhibitor therapy. Front Pharmacol 8: 49, 2017.

15. Postow MA, Callahan MK and Wolchok JD: Immune checkpoint blockade in cancer therapy. J Clin Oncol 33: 1974-1982, 2015.

16. Postow MA, Sidlow R and Hellman MD: Immune-related adverse events associated with immune checkpoint blockade. N Engl J Med 378: 158-168, 2018.

17. Haanen JBAG, Carbonnel F, Robert C, Kerr KM, Peters S, Larkin $\mathrm{J}$ and Jordan K; ESMO Guidelines Committee: Management of toxicities from immunotherapy: ESMO Clinical Practice Guidelines for diagnosis, treatment and follow-up. Ann Oncol 28 (Suppl 4): iv119-iv142, 2017.

18. Ohrndorf S, Glimm AM, Burmester GR and Backhaus M: Musculoskeletal ultrasound scoring systems: Assessing disease activity and therapeutic response in rheumatoid arthritis. Int J Clin Rheumatol 6: 57-65, 2011.

19. Calabrese C, Kirchner E, Kontzias K, Velcheti V and Calabrese LH: Rheumatic immune-related adverse events of checkpoint therapy for cancer: Case series of a new nosological entity. RMD Open 3: e000412, 2017.

his work is licensed under a Creative Commons Attribution-NonCommercial-NoDerivatives 4.0 International (CC BY-NC-ND 4.0) License. 